\section{Dynamic tomographic studies of interscapular feline injection-site sarcoma: essential or useless practice?}

$1-7$

(C) The Author(s) 2017

Reprints and permissions:

sagepub.co.uk/journalsPermissions.nav DOI: $10.1177 / 1098612 \times 17717176$ journals.sagepub.com/home/jfms

This paper was handled and processed by the European Editorial Office (ISFM) for publication in JFMS

(\$SAGE

\author{
Maurizio Longo ${ }^{1,2}$, Davide Danilo Zani², Roberta Ferrari², \\ Jessica Bassi ${ }^{2}$, Maria Elena Andreis ${ }^{2}$, Damiano Stefanello², \\ Chiara Giudice ${ }^{2}$, Valeria Grieco², Tiziana Liuti ${ }^{1}$, Ian Handel ${ }^{1}$ \\ and Mauro Di Giancamillo²
}

\begin{abstract}
Objectives Feline injection-site sarcomas (FISSs) are soft tissue tumours typically characterised by an interscapular location and highly infiltrative behaviour. CT is considered the modality of choice for FISS staging and double positioning (dynamic approach) was reported to successfully detect the exact extent of infiltration into the muscles. The aim of the present study was to investigate the utility of the dynamic approach in feline patients referred for preoperative staging of interscapular FISS.

Methods Tumour volume estimates were compared between the ellipsoid and the semi-automated segmentation methods. Two radiologists blinded to the patient coding used images from each position to assess the extent of muscular infiltration. The distance between the neoplasm and the adjacent skeletal structures (scapulae, spinous processes) was recorded in both positions by a single radiologist.

Results Fifty-nine of 84 neoplasms invaded the adjacent muscular structures, with up to 15 muscles infiltrated. Between the extended and flexed position the average estimated numbers of muscles infiltrated were 1.9 (extended) and 1.84 (flexed) for observer A and 1.89 (extended) and 1.85 (flexed) for observer B. Good agreement between observers was established, with higher tumour volumes detected via the ellipsoid method. Moreover, tumours with smaller volumes showed slightly decreased muscular infiltration. Marked difference in the recorded distance between the skeletal structures and the neoplasm in the two different positions was established (mean \pm SD difference spinous processes: $9.74 \pm 9.57 \mathrm{~mm}$; mean \pm SD difference scapulae $15.15 \pm 11.76 \mathrm{~mm}$ ).

Conclusions and relevance A dynamic approach should be used for a complete evaluation of the invasiveness of FISS along with appropriate methodology for tumour volume measurement, which could potentially alter the tomographic estimation of the real dimension of the neoplasms.
\end{abstract}

Accepted: 1 June 2017

\section{Introduction}

Feline injection-site sarcomas (FISSs) are highly infiltrative mesenchymal tumours that exhibit aggressive local behaviour in the adjacent musculoskeletal structures, such as the interscapular muscles, the spinous processes and the scapulae. The high local recurrence rate is one of the major concerns regarding this subcutaneous sarcoma, which is frequently located in the interscapular region. Even following the current surgical protocol of excising up to $3-5 \mathrm{~cm}$ of perilesional tissue and up to two muscles layers, the local recurrence rate is up to $14-22 \%$
${ }^{1}$ Hospital for Small Animals, Royal (Dick) School of Veterinary Studies, University of Edinburgh, Easter Bush Veterinary Centre, Roslin, UK

2Department of Veterinary Medicine, University of Milan, Milan, Italy

\section{Corresponding author:}

Maurizio Longo DVM, PhD, MRCVS, Hospital for Small Animals, Royal (Dick) School of Veterinary Studies, University of Edinburgh, Easter Bush Veterinary Centre, Roslin EH25 9RG, UK Email: mlongo@exseed.ed.ac.uk 


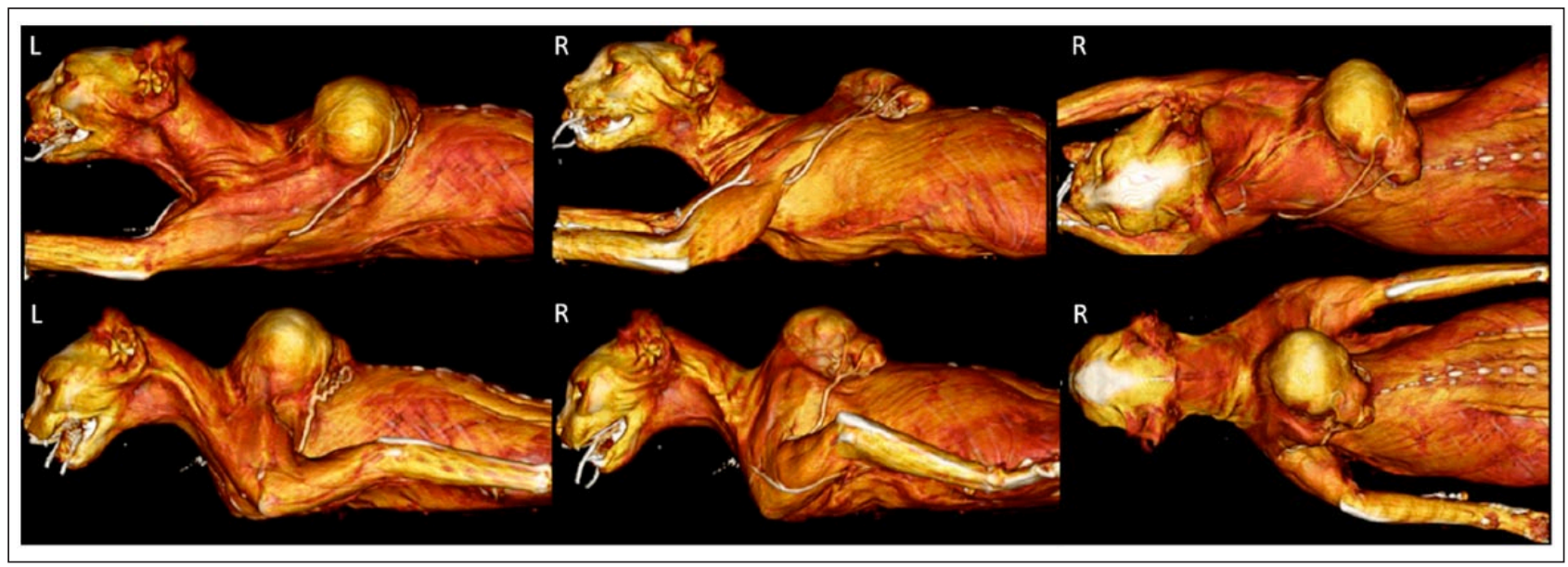

Figure 1 Three-dimensional CT multiplanar reformation reconstruction of the double positioning of a patient with the forelimbs extended cranially and flexed caudally along the body

in the case of non-infiltrated margins upon histopathological examination and up to $69 \%$ with infiltrated margins. ${ }^{1-5}$

Despite several different studies having focused on strategies for reducing the high local recurrence rate, including the use of chemotherapy and radiotherapy as useful adjuvant tools to reduce tumour regrowth, 2,3,6 and infiltration of the margins, this peculiar feature of FISS is still a relevant topic, because surgery is often the only therapy applied. ${ }^{2} \mathrm{CT}$ and MRI were introduced into clinical planning to better define the relationship between neoplastic lesions and the surrounding soft and hard tissues, considering the highly invasive nature of surgical excision..$^{7-9}$ Nonetheless, no specific gold standard diagnostic imaging technique was ever standardised for surgical planning in FISS cases, and even purely clinical evaluation of the neoplasm was proposed in the past as a sufficient basis for surgical planning. ${ }^{1} \mathrm{CT}$ is commonly applied to achieve a better understanding of the relationship with the adjacent musculoskeletal structures and for assessment of the deep margins. . $^{8,10}$ There are few published studies concerning new imaging strategies for specifically defining the anatomy of the region, ${ }^{11}$ and consequently its margins, or for computing the real volume of the neoplasm, which increase the agreement with histopathological measurements.10,12 Moreover, the effects of formalin fixation on the assessment of tumour volume were investigated by comparing CT measurements with histopathology. ${ }^{12}$ The results showed slight shrinkage of the FISS after excision and formalin fixation. The tumour volume estimated by three-dimensional (3D) software was described to be more accurate than the results of the ellipsoid formula, suggesting that the former method should be used when accurate estimation of tumour volume is particularly challenging on $\mathrm{CT} .{ }^{12}$
In 2013, it was postulated that as a consequence of the high mobility of the interscapular region, a common location for FISS, ${ }^{2}$ the relationship between the mass and the surrounding tissues may change depending on the position of the forelimbs during the CT examination. ${ }^{8} \mathrm{An}$ additional post-contrast scan with the forelimbs flexed along the body was suggested, after a conventional study with the forelimbs extended cranially (Figure 1), to allow correct estimation of the extent of local invasion and to avoid unnecessary invasive surgical resection of structures not infiltrated by the tumour, such as the scapulae or the spinous processes. ${ }^{8}$ The results of dynamic positioning in 10 patients with FISSs showed slightly reduced estimates of the number of muscles infiltrated by the tumour when the site was imaged in a flexed position. Therefore, double positioning (Figure 1) was suggested as a standard protocol for the evaluation of FISS when it is in an interscapular location. ${ }^{8}$

Nevertheless, there is a lack of more extensive case series highlighting, with convincing statistical evidence, the pros and cons of double positioning for estimating the number of structures infiltrated by an FISS. Thus, our first aim was to collect a high number of feline patients with interscapular FISSs to investigate the utility of double positioning in the preoperative evaluation of the neoplasms and the feasibility of its consistent application by different observers.

Moreover, we hypothesised that the volumes of the neoplasms could have a proportional impact on the musculoskeletal infiltration detected by $\mathrm{CT}$, with small neoplasms showing poor infiltration within the surrounding anatomical structures.

\section{Materials and methods}

Client-owned cats that underwent a CT examination for preoperative staging between December 2006 and 


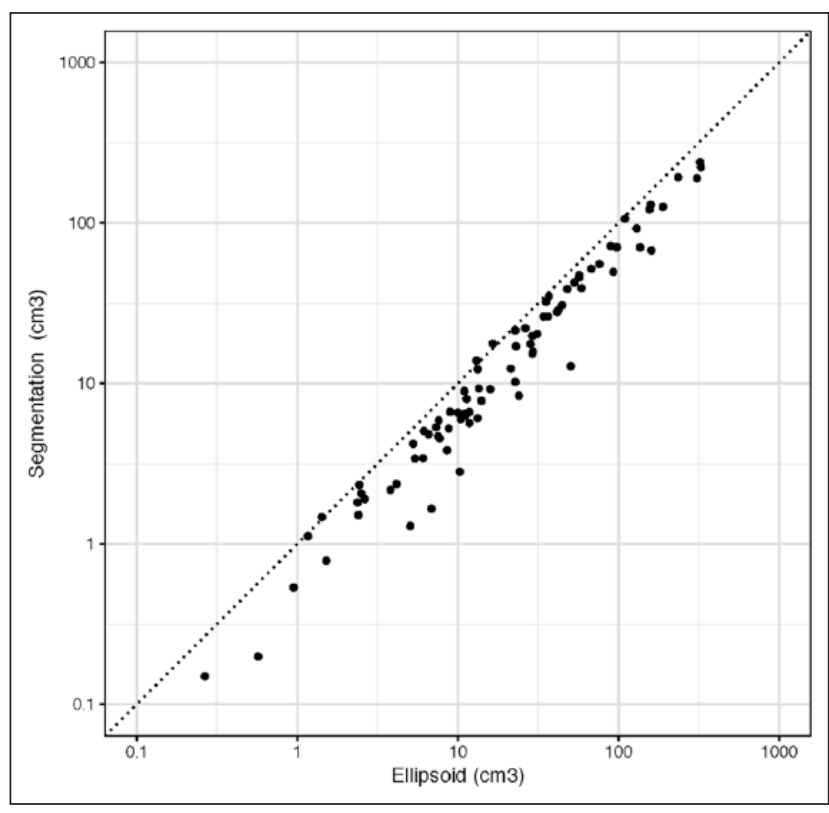

Figure 2 Scatter plot of ellipsoid estimates and segmentation estimates of tumour volume. The dotted line is an identity line. Logarithmic scales are used, as there was a wide range of tumour volumes. The ellipsoid method generally estimated higher neoplasm volumes than the segmentation method

January 2016 were included in the study if histological diagnosis of injection-site sarcomas was available. Only FISSs at first presentation and located within the interscapular region (between C5 and T5) were included. All the owners provided written consent before the diagnostic procedures.

CT studies were performed with two different scanners: a single-slice Picker PQ $2000 \mathrm{~S}$ (Philips) and a 16-slice GE Brightspeed Elite (General Electric). Images were acquired in helical mode with a thin slice thickness ranging between 1 and $3 \mathrm{~mm}$ and a pitch of 1 . Three thoracic and abdominal scans were performed with the patient in sternal recumbency: pre- and post-contrast scans with the forelimbs extended cranially and a final post-contrast scan with the forelimbs flexed caudally, along the body (Figure 1). Contrast medium (350 mgI/ $\mathrm{ml}$ Iohexol [Omnipaque; General Electric]) was injected at a concentration of $600 \mathrm{mgI} / \mathrm{kg}$ through the cephalic vein, either manually or with a power injector system (Medrad Mark V Plus; Bayer), at a rate of $2-3 \mathrm{ml} / \mathrm{s}$ according to the patient's weight.

The images were sent to PACS (Picture Archive and Communication System) and processed with certified medical software (OsirixPRO 64 Bit; Aycan Medical Systems). Two radiologists who were blinded to the patient coding assessed the number of muscles infiltrated by the tumour on the post-contrast scan in the extended and flexed positions, as previously described. ${ }^{8}$ Additional measurements consisted of tumour volume measurement using the ellipsoid formula $(4 / 3 \pi$ $\left.\left[1 / 2 X^{*} 1 / 2 Y^{*} 1 / 2 Z\right]\right),{ }^{10}$ where $X=$ width (laterolateral dimension), $\mathrm{Y}=$ height (dorsoventral dimension) and $\mathrm{Z}=$ length (craniocaudal dimension) and 3D semiautomated segmentation, similar to what has previously been reported in the literature. ${ }^{12}$ This measurement was performed by adjusting the window levels and computing the volume on the post-contrast scan using the 'ROI segmentation' tool provided in the certified medical software: an ROI (region of interest) was drawn around the tumour in every slice and then the software computed a volume-rendered model of the neoplasm. One radiologist recorded the distance between the neoplasm and the adjacent skeletal structures (spinous processes, scapulae) for all the patients, in both flexed and extended position. In each patient the widest difference of the distance between the spinous processes neoplasm and the scapulae neoplasm between the two positions was established. Absolute means and SDs of the measured differences in the distance from the spinous processes and the scapulae were calculated.

The results were recorded in a spreadsheet file. The $R$ statistical program (www.r-project.org) was used to plot and analyse the data. The relationship between ellipsoid volume and segmentation volume was assessed using a linear model with no intercept term, as a tumour with zero volume would be expected to have zero volume by either method. The difference in counts of muscle infiltration between the extended and the flexed positions was assessed using a two-sample permutation test with the null hypothesis that the distribution of counts was the same for the two methods. The observer discrepancy was assessed relative to the positioning method using an exact signed-rank test. The correlation between tumour volume and number of muscles infiltrated was assessed via Spearman's rank correlation.

\section{Results}

Among 248 cats referred for FISS staging, 84 cats met the inclusion criteria: 78 domestic shorthairs, two Chartreux, one Maine Coon, one Siamese, one Persian and one Norwegian Forest Cat. Their ages ranged between 1 and 15 years (mean 10.03 years, median 10 years). Weight was not available for 12 cats. For the remaining 72, mean and median weights were $4.4 \mathrm{~kg}$ and $4 \mathrm{~kg}$, respectively (range $2.8-7 \mathrm{~kg}$ ). The computed volumes ranged between 0.26 and $323.87 \mathrm{~cm}^{3}$ (average volume $45.9 \mathrm{~cm}^{3}$, median volume $16.41 \mathrm{~cm}^{3}$ ) with the ellipsoid formula and between 0.14 and $238.21 \mathrm{~cm}^{3}$ with the 3D software-based formula (average volume $32.24 \mathrm{~cm}^{3}$, median volume $10.18 \mathrm{~cm}^{3}$ ).

The relationships between the ellipsoid and the segmentation estimates of tumour volume are shown in Figure 2. A linear model of segmentation volume based on ellipsoid volume explained most of the variation in segmented volume (adjusted $\mathrm{r}^{2}=97.5 \%$ ). Segmentation 


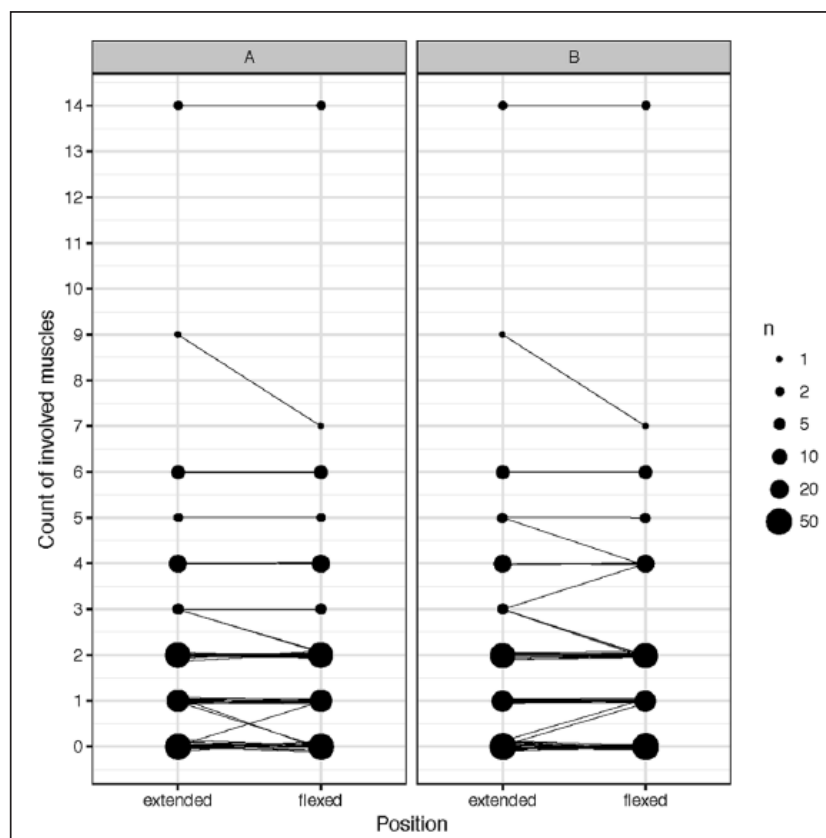

(a)
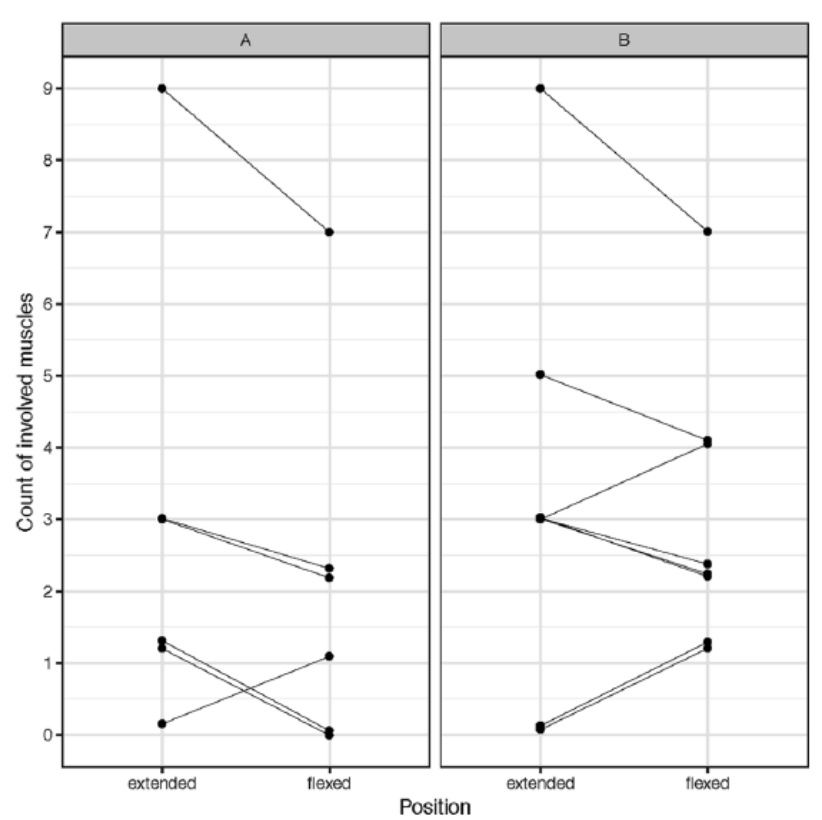

(b)

Figure 3 (a) Observer assessment of muscle involvement by positioning method. For each observer, assessments of the same tumour are joined by a solid line. The sizes of the points reflect the number of tumours assessed to have a particular count by each observer/positioning method. Horizontal lines represent assessments that were the same for both positions; sloping lines represent differing counts. (b) Subset of measurements from (a) where observers were internally discordant between extended and flexed measurements

volume was estimated to be $70 \%$ of ellipsoid volume (95\% confidence interval 67.5-72.3; $P<0.001$ ).

In total, 25 neoplasms did not show any relationship with the surrounding musculoskeletal structures, with increased adipose tissue lying between the neoplasms and the structures. The rest of the neoplasms $(n=59)$ invaded the adjacent muscular structures, with up to 15 muscles infiltrated. The most represented muscles were, in descending order of representation, the trapezius, rhomboid, latissimus dorsi, supraspinatus, longissimus colli, longissimus thoracis, dentatus dorsalis, dentatus ventralis, subscapularis, infraspinatus and scalenus.

Between the two positions (extended and flexed), the average estimated numbers of muscles infiltrated were 1.9 (extended) and 1.84 (flexed) for observer $\mathrm{A}$, and 1.89 (extended) and 1.85 (flexed) for observer B.

In six cases for observer A and eight cases for observer $\mathrm{B}$, a discrepancy between the extended and the flexed positions was detected. In five cases, observer A recorded higher muscular infiltration in the extended position, whereas in only one case did the same observer record a higher number of infiltrated muscles in the flexed position. For observer B, eight discrepancies were identified between the different positions, with five cases yielding a higher number of muscles infiltrated in the extended position and three cases in the flexed position. There was no evidence of a statistically significant difference between the extended and flexed counts of muscle infiltration ( $P=0.8708$, two-sample permutation test). Furthermore, there was no evidence of an inter-rater difference associated with the extended position compared with the flexed positions ( $P=0.6875$, exact signed-rank test) (Figure 3$)$.

The observers disagreed on five extended measurements and three flexed measurements. The overall disagreement between observers consisted of a difference of only 1 in the count of muscles infiltrated (Figure 3a,b).

There was a weak positive correlation between tumour volume and the number of muscles infiltrated by the tumour for both the extended (correlation $=0.52$; $P<0.001$ ) and the flexed (correlation $=0.51 ; P<0.001$ ) positions. No large tumours showed a complete absence of muscle infiltration; however, certain small tumours showed large amounts of infiltration (Figure 4).

In 38 cases, the FISS appeared to be adjacent to at least one spinous process, with only one case showing mixed osteolytic and osteoproliferative changes. Of 112 spinous processes appearing adjacent to the FISS, 84 showed similar adhesions in both positions, 19 were adjacent to the FISS in the flexed but not in the extended position and nine were adjacent in the extended but not in the flexed position.

Contiguity of the tumour to at least one scapula was visible in 24 cases, with a total of 32 scapulae close to the FISS: 15 were adjacent in both positions, 12 only in 


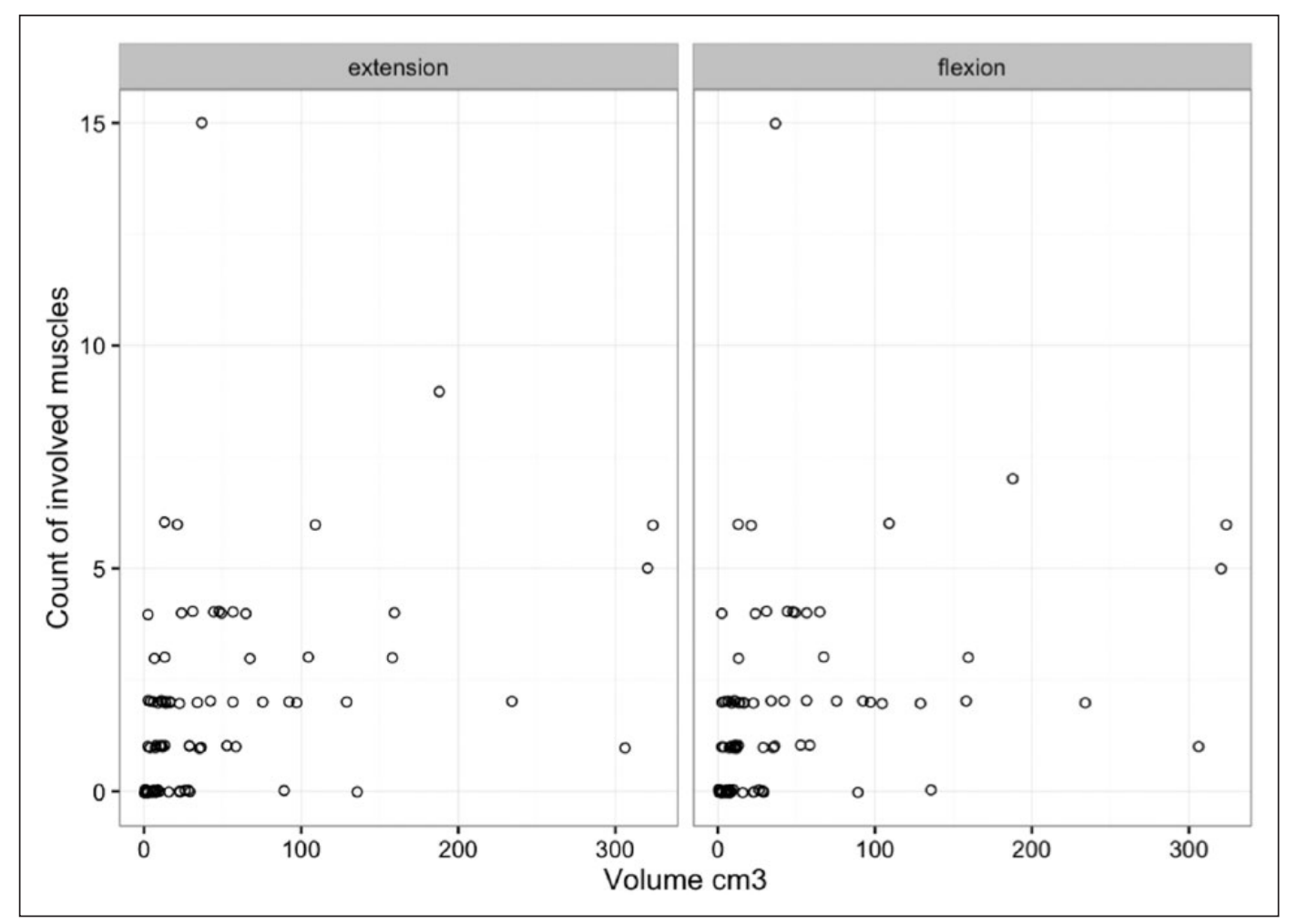

Figure 4 Scatter plot showing the count of muscles involved vs tumour volume for both positioning methods. The points were adjusted vertically to separate them for visibility. Higher muscle infiltration is visible at higher volumes; however, a few small neoplasms show consistent infiltration

flexion and five only in extension. No bone changes were detected at the level of the scapulae. The distance between the neoplasm and the spinous processes ranged between 0 and $60 \mathrm{~mm}$ in extension and between 0 and 26 $\mathrm{mm}$ in flexion. The distance between the neoplasm and the scapulae ranged between 0 and $55 \mathrm{~mm}$ in extension and between 0 and $100 \mathrm{~mm}$ in flexion. The absolute mean \pm SD difference in the distance between the spinous processes neoplasm in both positions was 9.74 $\pm 9.57 \mathrm{~mm}$, whereas the absolute mean difference between the scapulae neoplasm was $15.15 \pm 11.76 \mathrm{~mm}$.

\section{Discussion}

FISSs are fast-growing tumours characterised by local infiltration towards the musculoskeletal structures and a high local recurrence rate (19-69\%), ${ }^{1,3,4}$ whereas the distant local metastatic spread is reported to be considerably low, between $12 \%$ and $28 \%{ }^{1,3,13}$ These peculiar features of the tumour make surgical planning an essential step to achieve wide and/or radical excision of the neoplasm, which may provide a longer disease-free period (901 days) than excision of neoplasms with infiltrated margins (79 days). ${ }^{1,3}$ CT detection of the peripheral digitations, including tumour extension, skip metastasis and perilesional panniculitis, is of critical importance for scar tissue healing and reduction of tumour regrowth.

The surgical goal is the removal of as much perilesional tissue as possible, and CT detection of the peripheral digitations, including tumour extension, skip metastasis or perilesional panniculitis, can help to direct surgeons' preoperative evaluation. ${ }^{1,2,4,8}$ However, because of the highly dynamic interscapular location of the tumour, the adjacent skeletal structures could appear to be involved based on conventional $\mathrm{CT}$ images acquired with the forelimbs extended cranially (Figure 1), resulting in highly aggressive surgery for resection of the desired amount of perilesional marginal tissues. ${ }^{8}$

In 2013, Travetti et al first described the dynamic approach, ${ }^{8}$ with results from 10 feline patients affected by interscapular FISS preliminarily showing a slight reduction in the estimate of muscular infiltration by the tumour between flexed and extended positioning of the forelimbs. In fact, the large amount of fat between the scapula and the fascial planes makes this region extremely movable, as shown in a previous publication describing the changes in relationship of the musculoskeletal structures between different imaging modalities (CT, MRI and gross sectional anatomy), ${ }^{11}$ using a dynamic approach in non-pathological patients. 
Although in the majority of our cases the number of infiltrated muscles was the same and the intra- and inter-rater analysis did not demonstrate any statistically significant difference between extended vs flexed positioning, slight discrepancies between the two positions and between observers could be descriptively observed (six for observer A, eight for observer B). It is possible that the limited number of cases with muscle infiltration (59/84 subjects) and with a muscle infiltration number $>1$ (41/59 cases with muscle infiltration) could have influenced the statistical results. Moreover, there were five cases in which both observers recorded fewer infiltrated muscles in limb flexion than in extension and this is in accordance with what has previously been suggested. ${ }^{8}$ The discrepancies in measurements between the observers could be considered unrelated to the two different positions (Figure 3a,b).

Our results partially agree with the 2013 study by Travetti et $a l, 8$ strongly suggesting that veterinarians consider double positioning for a correct estimation of FISS, although it is difficult to choose the most accurate positioning for studying FISS. We believe that thorough estimation of a tumour's margins and infiltration could potentially decrease unnecessary inclusion within the perilesional margins of structures not involved in the pathological process. Moreover, the measurements of the distance between the FISS and the adjacent skeletal structures (vertebral spinous processes, scapulae) were markedly different between the two positions, with a mean difference of $9.74 \mathrm{~mm}$ for the spinous processes and $15.15 \mathrm{~mm}$ for the scapulae. It means that structures at the edge of perilesional margins of excision could potentially be included or not into the desired margins, depending on the position acquired by the patient. In a previous paper no measurements of the changes between the skeletal structures adjacent to the neoplasms were quantified. ${ }^{8}$ This result also supports the inclusion of an additional dynamic evaluation with the forelimb flexed caudally along the body (Figure 1) when the neoplasm is adjacent to the skeletal structures, to underline possible movement of the neoplasm away from these structures. In fact, movement of these structures away from the neoplasm could refine the amount of tissue included in the perilesional margins based on the CT evaluation, especially in cases in which a reduced amount of subcutaneous adipose tissue leaves the interscapular structures in close proximity (Figure 1).

The assessment of tumour dimensions using a clinical calliper has shown wide limits of agreement compared with CT, with increased variability for neoplasms with increased size. ${ }^{10}$ Both CT and MRI were described as useful tools for the investigation of FISS; ${ }^{7-9}$ however, CT is commonly used because of its ability to characterise and stage a tumour within the same scan. ${ }^{8-10}$ Moreover, multiplanar reconstructions represent an important advantage for the correct estimation of tumour volume, reducing the gap between tomographic volumetric measurements and histopathology.

We tested the difference in the volumes computed with both a semi-segmentation software tool and the ellipsoid formula. Our results show considerably different measurements between the two-dimensional and the 3D methods (Figure 2). In particular, in contrast to what has been stated by others, ${ }^{14}$ the tumour volume estimated by the ellipsoid formula was consistently significantly higher than that estimated by the 3D CT software tool (Figure 2). These results could be attributed to the following; first, several of the neoplasms included in the sample showed a very irregular shape, the size of which could be easily overestimated by the ellipsoid formula and less so by the semi-automated computed volume measurement, where the contour of the mass is exactly drawn manually and computed by the algorithmic conversion. Second, the software used here was different from that previously described, which consisted of fully automated segmentation. However, we consider this second possibility less likely because in the entire sample population no variation was recorded in the results, and, in general, it is more likely that the assumption of an ellipsoid shape could cause a slight overestimation of tumour volume. The reduced sample size in the previously reported study could potentially have affected the results if less irregular neoplasms were present.

In humans, the application of accurate segmentation is crucial on MRI, CT and ultrasound when assessing a tumour using RECIST (Response Evaluation Criteria in Solid Tumours $)^{15}$ or the progression of diseases such as benign prostatic hyperplasia. ${ }^{16}$ Semi-automated and fully automated techniques produce reliable exact measurements of the pathological tissue and will be considered essential in the future, when ultrasound-CT, positron emission tomography (PET)-CT and PET-MRI fusion techniques will be widely applied in veterinary medicine.

In the present study, the close concordance between the two radiologists in estimating muscular infiltration resulted in only eight disagreements among 84 cases; this low rate of disagreement likely resulted from the similar advanced degree of experience of the two observers. A weak correlation was detected between tumour volume and the number of infiltrated muscles: as expected, infiltration was higher with larger volumes. However, caution should be taken because even a few small tumours showed consistent muscular infiltration (up to 15 muscles; Figure 4). These results are in agreement with previous postulations and confirm that even small neoplasms can show highly infiltrative behaviour towards the surrounding musculoskeletal structures. ${ }^{16}$ In the light of this possibility, we suggest that veterinarians consider a complete diagnostic imaging work-up even in the case of a small palpable lesion to avoid incorrect assessment of the neoplastic infiltration. 
Based on our results, neither position was always more accurate than the other; however, the flexed position reached a lower infiltration rate in five patients between the two observers. It is our opinion that the flexed position should be added to the conventional extended one to highlight any difference in muscle infiltration. Musculoskeletal structures that are consistently infiltrated in both positions are more likely to represent neoplastic invasion. Hence, we recommend excision of any structure that is consistently adjacent to the FISS in both positions.

One main limitation is the lack of information on postoperative and oncological outcomes for the patients included in this study that could highlight the real benefit of the dynamic approach. Future investigations are warranted to understand how CT diagnostic performance will affect the clinical outcome of patients affected by FISSs, considering the effects of the different excised margins between positions. An additional limitation of this study is the increased amount of radiation exposure due to the additional final scan, although we consider this aspect of less concern for veterinary oncological patients.

\section{Conclusions}

Our study strongly suggests that veterinarians should consider a dynamic CT approach in the study of interscapular FISS by including an additional post-contrast scan with the forelimbs flexed caudally along the body when the tumour lies in the interscapular region, in proximity to the musculoskeletal structures. Semiautomated segmentation of the neoplasms should be used for accurate preoperative planning of tumour volume, including in the case of small neoplasms that could potentially show highly infiltrative behaviour on CT.

Conflict of interest The authors declared no potential conflicts of interest with respect to the research, authorship, and/ or publication of this article.

Funding The authors received no financial support for the research, authorship, and/or publication of this article.

\section{References}

1 Phelps HA, Kuntz CA, Milner RJ, et al. Radical excision with five-centimeter margins for treatment of feline injection-site sarcomas: 91 cases (1998-2002). J Am Vet Med Assoc 2011; 239: 97-106.

2 Martano M, Morello E and Buracco P. Feline injection-site sarcoma: past, present and future perspectives. Vet J 2011; 188: 136-141.
3 Bray J and Polton G. Neoadjuvant and adjuvant chemotherapy combined with anatomical resection of feline injection-site sarcoma: results in $\mathbf{2 1}$ cats. Vet Comp Oncol 2014; 14: 147-160.

4 Cantatore M, Ferrari R, Boracchi P, et al. Factors influencing wound healing complications after wide excision of injection site sarcomas of the trunk of cats. Vet Surg 2014; 43: 783-790.

5 Giudice C, Stefanello D, Sala M, et al. Feline injection-site sarcoma: recurrence, tumour grading and surgical margin status evaluated using the three-dimensional histological technique. Vet J 2010; 186: 84-88.

6 Ladlow J. Injection site-associated sarcoma in the cat. Treatment recommendations and results to date. J Feline Med Surg 2013; 15: 409-418.

7 Rousset N, Holmes MA, Caine A, et al. Clinical and lowfield MRI characteristics of injection site sarcoma in 19 cats. Vet Radiol Ultrasound 2013; 54: 623-629.

8 Travetti O, Di Giancamillo M, Stefanello D, et al. Computed tomography characteristics of fibrosarcoma - a histological subtype of feline injection-site sarcoma. J Feline Med Surg 2013; 15: 488-493.

9 McEntee MC and Samii VF. The utility of contrast enhanced computed tomography in feline vaccine associated sarcomas: 35 cases [abstract]. Vet Radiol Ultrasound 2000; 41: 575 .

10 Ferrari R, Di Giancamillo M, Stefanello D, et al. Clinical and computed tomography tumour dimension assessments for planning wide excision of injection site sarcomas in cats: how strong is the agreement? Vet Comp Oncol 2017; 15: 374-382.

11 Longo M, Modina SC, Bellotti A, et al. Advances in the anatomic study of the interscapular region of the cat. BMC Vet Res 2015; 11: 249.

12 Terry JL, Milovancev M, Lohr CV, et al. Changes in the dimension and volume of feline injection-site sarcomas following formalin fixation as determined by use of the ellipsoid volume formula and three-dimensional computed tomography software. Am J Vet Res 2016; 77: 620-626.

13 Romanelli G, Marconato L, Olivero D, et al. Analysis of prognostic factors associated with injection-site sarcomas in cats: 57 cases (2001-2007). J Am Vet Med Assoc 2008; 232: 1193-1199.

14 Travetti O, Stefanello D, Zecconi A, et al. The relationship between tumor volume and local invasiveness in feline injection-site sarcoma: a retrospective CT study on 200 cases [abstract]. Vet Radiol Ultrasound 2011; 52: 230.

15 Hayes SA, Pietanza MC, O'Driscoli D, et al. Comparison of CT volumetric measurement with RECIST response in patients with lung cancer. Eur J Radiol 2016; 85: 524-533.

16 Garvey B, Turkbey O, Truong $\mathrm{H}$, et al. Clinical value of prostate segmentation and volume determination on MRI in benign prostatic hyperplasia. Diagn Interv Radiol 2014; 20: 229-233. 